\title{
EVIDENCE-DRIVEN POLICY MAKING USING HETEROGENEOUS SOURCES OF DATA - THE CASE OF A CONTROL PARKING SYSTEM
}

\author{
Anastasios Papazoglou Chalikias ${ }^{1}$, Ioannis Tsampoulatidis ${ }^{1,2}$, Filareti Tsalakanidou, \\ Spiros Nikolopoulos ${ }^{1}$, Ioannis (Yiannis) Kompatsiaris ${ }^{1}{ }^{1}$ Konstanstinos Doudouliakis ${ }^{3}$, \\ Petros Papafilis ${ }^{3}$ and Georgios Papastergios ${ }^{3}$ \\ ${ }^{\text {I CERTH-ITI, Greece }}$ \\ ${ }^{2}$ Aristotle University of Thessaloniki, School of Architecture, Greece \\ ${ }^{3}$ Municipality of Thessaloniki, Greece
}

\begin{abstract}
Policy making is generally and largely based on intuition and not backed up by evidence. Our goal is to transform the existing paradigm of policy making into an evidence-driven approach enabled by heterogenous sources of data. Methods for data collection, efficient storage and data analysis are implemented to measure the economic activity, assess the environmental impact and evaluate the social consequences of certain policy decisions. The extracted pieces of evidence are used to inform, advise, monitor, evaluate and revise the decisions made by policy planners. In this paper, we examine the use case of Thessaloniki's controlled parking system and its optimization balancing between economic growth, environmental impact and citizen's satisfaction, by harmonizing and visualizing heterogenous sources of data. The case study evaluation consists of a multi-step process that involves user recruitment, user training, formulation of platform-usage scenarios, platform evaluation based on questionnaires, and finally in-depth questionnaire analysis.
\end{abstract}

\section{KEYWORDS}

Policy Making, Big Data, Visualization, Economic Growth, Environmental Impact, Citizen Satisfaction

\section{INTRODUCTION}

In the past years, interest in the study of policy making has been on the increase. Large multimodal datasets are increasingly available on some facets of policy making and a few useful concepts have been developed, as comprehensibly described in (Dunn, 1981). While some countries have engaged in interesting improvement experiments, no shift for the better in overall policy making has occurred (Dror, 2017). A big issue with the current paradigm of policy making is that there is not enough evidence to justify their creation, thus depending on intuition.

As stated in (Sanderson, 2002) there are two main forms of evidence required in the approach to improve governmental effectiveness. The first is evidence to promote accountability in terms of results - evidence that the government is working effectively. The second is evidence to promote improvement through more effective policies and programmes - evidence of how well such policies and programmes 'work' in different circumstances. These two forms of evidence are different in nature. The first is primarily in the form of information on attributes of performance and is reflected in the growth of performance management in government (Treasury, H. M. S., 2000). The second form of evidence is qualitatively different from the first and is concerned with the knowledge of how policy interventions achieve change in social systems. In the notion of a modern government, evaluation in particular is required to play an enhanced role in providing information on performance to enhance accountability and 'control by results', and in providing evidence of what works to inform policy learning and improvement.

The overall purpose of this article is to explore the notion of evidence-based policy making in the context of policy intervention in increasingly complex social systems and the potential role of piloting and evaluation. Instead of only relying on evaluation during or after the piloting phase after a specific policy is in place, we try to transform the existing paradigm of policy making into an evidence-driven approach aided by 
the proactive use of heterogeneous sources of data so as to justify every single policy approach. This means that the evaluation of a specific policy can be performed even before a policy is in place.

Our ambition is to differentiate from existing approaches by simultaneously considering environmental, economic and social data, enabling in this way the support of policy design in a holistic manner, taking into account urban resilience. In addition, the proposed approach enables the meaningful cross-correlation of different indicators that can allow shifting from simple observations to short-term predictions. We propose an approach to homogenize the heterogeneous sources of data by using their spatio-temporal properties to interconnect them. In this way, we can support complex queries and facilitate decision making processes that need to simultaneously evaluate the impact of more than one aspects (i.e. environmental, economic and social).

\section{METHODOLOGY}

\subsection{Multi-faceted Approach for Policy Design, Implementation and Evaluation}

Our proposed methodology foresees the use of heterogenous data sources to facilitate the decisions required to reach the strategic goals of the city of Thessaloniki. In facilitating this process, we adopted the IAMER (Inform - Advise - Monitor - Evaluate - Revise) approach for developing a multi-faceted dashboard. This dashboard is composed of five different facets that present information and extract insights that are essential to the decision-making process. More specifically: a) Inform: Extracts and presents the evidence that are necessary for policy makers to make informed decisions when defining the projects, policies and programs incorporated in their technical program; b) Advise: Presents the decision makers with the estimated impact on their planned activities on the resiliency status of their city; c) Monitor: Relies on the data obtained from the sensing infrastructures and use the data mining and visualization tools to establish a set of monitoring mechanisms; d) Evaluate: Building on the information gathered through the monitoring mechanisms defines a set of KPIs (Key Performance Indicators) that allows quantifying and assessing the impact of the enforced policies. These KPIs serve as the criteria for success or failure of the enforced policies; e) Revise: Depending on the value of the KPIs revises the projects, policies and programs for next years ' technical program considering also the weaknesses that have caused certain policies to fail. Our main focus in this work lies on the first two facets of the IAMER methodology, Inform and Advise.

\subsection{Architecture}

Our platform's architecture, consists of seven incremental layers that establish a powerful Software as a Services (SaaS) solution for city data science in the direction of urban development.

Data Layer: There are three main categories of data that are used in our platform: i) Real-time data coming from sensors such as vehicle emission measurements, but also historical data found in archives to form time-series data. These data are used in the analytics layer to sense the environmental impact. ii) Structured data coming from official sources e.g. GIS-based data on income from a car parking system. These data will be used in the analytics layer to sense the economic activity. iii) Unstructured data coming from the open participatory platforms, making engaged citizens to act as living sensors. These data are used in the analytics layer to sense the social consequences.

Collection Layer: The successful collection, integration and analysis of the data classified under the above categories play a significant role. Having in mind the extent of data heterogeneity, our platform integrates the collected data under a common analysis framework. Several pre-processing activities towards data cleaning and harmonization are performed according to (CUTLER Deliverable 3.2). During the data cleaning process, data errors are classified into four types: i) Pattern violations: The data are transformed to deal with formatting or attribute value issues. ii) Rule violations: Values violating any kind of integrity constraints, for example unique value fields inside data entries. iii) Duplicates: Filtering of duplicate entries. iv) Outliers: Outlier detection using analytical methods and techniques tailored to each case.

Storage Layer: Our platform's secure large scale storage and processing infrastructure is based on a hybrid cloud based approach. The focus is on the design of a flexible and horizontally scalable architecture that can be easily customized. The solution leverages open source technologies as well as proprietary ones. The architecture takes a security-by-design approach to address any significant threats identified in the threat analysis (CUTLER Deliverable 2.2). The cloud based and network infrastructure, provides a highly scalable 
bandwidth and capacity that would facilitate the extensive scope of advanced development and use case scenario testing.

Analytics: The purpose of this layer is to translate information into evidence and actionable knowledge by utilizing data analytics. This layer consists of algorithms that operate and aggregate time-series data, interconnect and filter data on a spatio-temporal level, as well as analyze data using machine learning algorithms (e.g. sentiment analysis on textual data provided by citizens in participatory platforms).

Visualization: The platform is designed to act as a multi-tenant, cloud-based, advanced data analytics tool for policy makers and is deployed in a multi-facet dashboard. The visualization widgets allow policy makers to customize the software based on their own needs and requirements. The visualization widgets are coming from economic, environmental and social aspects and builds upon visualization methods such as clustering maps, various charts, tag clouds, and others.

Policies: The outcome and integration of the above layers forms our ecosystem which purpose is to facilitate policy design using heterogenous sources of data towards urban development that effectively balance between economic growth, environmental impact and social consequences. Using this ecosystem the city officials are able to carry out a full cycle of policy development process, including design, implementation and evaluation.

\subsection{Data Types and Harmonization}

Data harmonization refers to the process of aligning data from heterogeneous sources into a coherent and unambiguous set (CUTLER Deliverable 3.2). Therefore, to deal with various naming and structural differences among diverse data sources, we have relied on the following rules: i) Time: Date and time information within have different naming and representations. It was decided to use the following naming conventions where present: Time, Date, DateTime, Timestamp, Year, Month, WeekDay; ii) Geographical: Geographic information, is mostly provided as geographical coordinates, but sometimes it is also given at the level of territorial units, e.g. municipalities. It was decided to follow the approach suggested by Nomenclature of territorial units for statistics (NUTS). If geographical coordinates are provided, then it is recommended to use "latitude", "longitude", and "altitude" naming accepting decimal values; iii) Environmental: A unified schema was established to model each parameter in terms of naming, units, timezone and usage of spatial information; iv) Economic: Most data regarding economic activity are collected by a central statistics office. The collected data then become available to international organizations like the Organization of Economic Cooperation and Development (OECD) and Eurostat among others. Thus, the variables' names, the measurement units and all other reporting indicators are identical across these data sources; v) Social: Considering the heterogeneity of social data sources, it is necessary to define a unified data model, so that the data is harmonized before processing. The data model that we have used in our approach is an extension of the SIMMO (Socially Interconnected MultiMedia-enriched Objects) model.

Moreover, the need to take into account different aspects of a certain policy (i.e. economic growth, environmental impact and social consequences) calls for an approach that would enable to query and interact with heterogeneous sources of data in a seamless manner. In this direction, all sources of data need to be tightly interconnected so as to enable their dynamic filtering and interactive interrogation. To this end, we have relied on the spatio-temporal properties of the data to interconnect them. More specifically, we are harmonizing our heterogenous data sources by using various techniques to make sure that each one of them have a spatio-temporal dimension. This way the different datasets can be projected in the same space and we can find correlations between them that impact the envisioned policies. By adding a longitudinal dimension to our datasets that are lacking one, allows us to test new hypotheses about urban structure and dynamics, and by taking into account the specific timeframes that these data belong to, we can predict with certainty specific recurring events.

\section{POLICY}

\subsection{Controlled Parking System}

Thessaloniki is a densely inhabited city with limited space available for building new infrastructure. A way of providing solutions to continuing urban problems is to change the use of the existing infrastructure. The area with most different uses is the 1st Municipal District, with people moving towards or away from it 
during day and night and there is a very high demand for parking spaces. Consequently, the available parking space needs to be controlled.

Since 2017, a new controlled parking system (CPS) has been installed in the 1st and 3rd Municipal Districts of Thessaloniki, which is known as THESi (THESi). The available parking space is divided into white and blue sectors. White sectors are destined for visitors while blue sectors are reserved for permanent residents. More specifically, every visitor must pay a fee per hour in order to park his vehicle in one of the spots of a white sector. At the same time, each resident has to go through an application process, submitting all necessary material/documentation to municipal authorities. This way he can become a holder of a parking card that will allow him to park his vehicle in any spot in a blue sector of his district. In each of the different White or Blue sectors there are multiple parking spots.

The system is supervised by the Municipal Police, which plays a crucial role in the establishment of the CPS. Whenever a new measure is imposed there is always dissatisfaction expressed by part of the population and a social risk needs to be taken into consideration. Visitors that do not pay a parking fee, visitors that park in blue sectors, permanent residents that do not apply for a parking card but persist to park in blue sectors, all have to receive penalty notices. In addition, there were complaints from residents that the parking spots are not enough, so the Municipality Department was confronted with the request to change the characterization of some parking spots from white to blue. Nevertheless, the selection of which parking spots to switch while taking into account economic growth, environmental impact and social consequences is a problem of combined optimization.

\subsection{Deciding on the Distribution of Parking Spots}

In deciding which parking spots need to be switched, there is a multitude of factors to be taken into account, including: a) Economic: Select parking spots that are under performing in terms of occupancy and generating revenue. b) Environmental: Select parking spots that are placed on streets exhibiting high levels of emissions. c) Social: Select parking spots in streets where there seems to be expressed dissatisfaction from citizens about illegal parking. In addition, select parking spots that exhibit an increased level of penalty notices that impacts both economic (loss of earning due to reduced parking fees) and social (delinquent behavior showing dissatisfaction).

The Municipal Police is providing data coming from daily patrols and data for penalty notices. The goal of using this data is to locate parking sectors with large numbers of penalty notices so as to propose possible changes in use (from visitors to residents) so as to reduce the number of penalty notices and increase citizens' satisfaction.

The Economic Department provides data about the daily income and occupancy of the parking spots. These data are used as evidence upon which changes in policy will be decided. For example, when deciding which sector should be switched from Visitor to Resident, the sector occupancy should be considered so as to minimize profit loss.

The Environmental Department is providing data for air pollution, obtained by existing monitoring stations. The data are processed to obtain values at street level, so there can be an overview of the air pollution footprint of the sectors and what will be the impact of a change in the distribution of sectors. A sector that belongs to a street with increased emissions should be preferred to switch into a residents sector, over a sector belonging to a street with low emissions. We work under the assumption that by changing a sector from visitor to resident, we reduce the associated traffic and thus the generated emissions.

The Improve My City platform (Improve My City) (Tsampoulatidis et al., 2013) is the citizen reporting tool the municipality of Thessaloniki is using to administer citizen issues. Using data from the aforementioned issues we can discover problems that citizens are experiencing in relation to the CPS, by recognizing user sentiment for a specific area and further filtering it down to street level.

\subsection{Data Sources}

A GeoJSON file (a file format for encoding a variety of geographical data structures) has been provided by the Municipality of Thessaloniki that features information about the entirety of the parking spots like, each sector spot area as a polygon, the address and Municipal District that the spots belong to, and their sector unique identification number. We use this file in conjunction with our data sources to cross correlate information between datasets and find a common dimension between them. More specifically the data sources are comprised of: 
Parking scans: The municipal police is patrolling the streets of Thessaloniki, where the CPS is in place. They are equipped with a device that reads the license plate of the scanned vehicle, checks the status of the vehicle and saves a multitude of data for each scan. The data include, a pair of GPS coordinates (latitude and longitude), a date time timestamp, the device id, and the result of the scan. Scan result can be: i) Legal: The car owner has paid for the parking spot and the parking duration hasn't exceeded the time limit period that he paid for. ii) Resident: The car owner is a resident of the area so he can park legally for free. iii) Illegal: The car owner is not a resident neither have paid for the specific spot. We calculate each parking scan entry GPS coordinates distance from each parking spot polygon centroid, using the distance() Turf.js (Turf.js) function that uses the Haversine formula. After we sort the entries based on the minimum distance value in meters we consider as match the first entry that its distance is less than 10 meters. Also we add to each entry additional sector information like its unique sector identification number and the Municipal District that it belongs to.

Parking spot income: From the economic department we have daily income data per spot, collected during the use of the parking system. This dataset includes a sector's address, number of parking spots, spot economic performance, and income per spot. After cleaning up the data we also add sector information for each entry like its identification number, the Municipal District that it belongs to, the street address and the sector type. Additionally for each parking sector we calculate the parking sector centroid and use it as GPS coordinates for location data for each entry.

Vehicle emissions: There are nitrous oxide (NOx) and non-methane volatile organic compound (NMVOC) emission data readings available about a number of major streets in Thessaloniki in 15 minute intervals. To be able to incorporate them into our dataset, we created new entries for each sector, so as to have more values down to sector level. This means that the total number of entries is all emission entries that are tied to a street multiplied with the number of the unique sectors that are inside this street. Moreover, we connected the emission entries to GPS coordinates calculating each sector's centroid and using it as GPS coordinates and added information about the Municipal District that it belongs to, as well as the sector type.

Improve My City data: To incorporate a social aspect into our dataset, we took advantage of the data contributed by citizens in Improve My City. By using this platform citizens submit their everyday issues through a webpage or a mobile application to the Municipality, and get back feedback on the expected resolution. A mechanism has been set up that indexes user issues to our platform, with information like the issue title, description and category that are useful for tag cloud creation, sentiment analysis and category filtering. Sentiment analysis has been performed on the textual description associated with each issue using the algorithm presented in (Tsakalidis et al., 2018). The issues also contain information that connects them to our previous datasets like street address, sector id, Municipal Area and GPS location. To calculate the GPS location coordinates we followed the same approach as in the parking scans. We calculated each issue's GPS coordinates distance from each parking spot polygon centroid, using the distance() Turf.js (Turf.js) function that uses the Haversine formula. After we sorted the entries based on the minimum distance value in meters we considered as match the first entry that its distance was less than 10 meters.

\section{USE CASE}

\subsection{Dashboard}

We have used Elasticsearch (Elasticsearch) to store and query our data, and Kibana (Kibana) to visualize them into charts inside a Dashboard. The Dashboard (CUTLER Thessaloniki pilot) that we have created has been set up and populated with charts from economic, social and environmental data. In this dashboard we focused on creating charts that contribute to easy decision making, when trying to decide which Visitor sector should change into a Resident one, minimizing economic turnover losses and vehicle emissions per street, while maximizing citizen satisfaction. Inside the dashboard a user can select a date range and since all sources of data are time bound, all charts update their content accordingly. The user can also select an area on any of the map charts that are available, performing a special query. Charts with the parking scans clustered all around the city, exist to give a quick overview on which sectors or streets are more frequently visited by the Municipal Police. The user can also see the vehicle emission data for a number of large streets and decide on which sector to select based on the emission street levels. Information about the economic performance of a specific sector and the distribution of scan types per sector, can also be observed so as to draw some conclusions on sector spots income. Extracted social data from the Improve My City platform, namely the description the users leave when they submit an issue to a specific street, are included in our dataset in the 
form of user sentiment using sentiment analysis. This way the user of the dashboard can recognize the citizen sentiment about a street or sector, and further analyze if it has any ties to the parking system or it is totally unrelated.

\subsection{Usage Scenario}

A usage scenario was created as a starting point for decision makers to use our dashboard as a policy making tool. In this concrete scenario, the Municipality of Thessaloniki is receiving complaints from residents about the insufficient number of parking spots and our platform is intended to help the decision makers to select the optimal sector for changing its use.

As we access the dashboard, in the chart shown in Figure 1.A there is a clustered view of the total parking scans performed to give an overview of how the administration patrols the sectors. Bigger circles denote larger scan frequency. In Figure 1.B, the user can click on a Municipal Area to select it and filter the data that belong only to this area. In our case, we want to see data only from the first Municipal Area, so we can click on Municipal Area 'A'. We can see that the changes reflect on all charts, so the dashboard is now refreshed to show only data from Municipal Area A.

Next we can see in the chart of Figure 1.C the Nitrous Oxide emissions for a number of large streets. Some streets have higher emissions than others, so hypothetically we can lower emissions if we focus our attention in sectors that are included in these streets. From the Street Selector component as shown in Figure 1.D we can select only the streets that have high Nitrous Oxide emissions to filter the dashboard accordingly, namely 'DRAGOUMI I.', 'VENIZELOU EL' and 'MITROPOLEOS'.

In Figure 1.E we can see the chart for the revenue / performance data for each sector. We can filter through the sectors that are located inside certain streets that are performing better than others. Thus, we can safely exclude streets we included in the previous step from our list of options since we want the sectors that are generating a high amount of revenue to remain intact. In our case we exclude the 'MITROPOLEOS' street, so we are left with 'DRAGOUMI I.' and 'VENIZELOU EL'.

Next we go to the social data charts illustrated in Figure 2.A, that are populated with data from the Improve My City platform, cross referenced with street addresses. There is a heatmap that depicts how many issues the residents of Thessaloniki submitted in this area, a Pie chart that shows a percentage for the categories of these issues, and a dropdown where you can filter the category that you want to further investigate. We select the illegal parking category from the dropdown so we can only see citizen issues that are related to parking. In the sentiment stacked bar chart as seen in Figure 2.B we can see the sentiment of the people extracted from the submitted issues. This way we can identify through the social aspect of the dataset, in which sectors the citizens have negative feelings on issues related to parking. So we can safely exclude streets that users are less negative about than others from the Street Selector dropdown chart that we used before to end up with one street. In our case we exclude street 'VENIZELOU EL.' and end up with 'DRAGOUMI I.'.

Going further down on the charts of the dashboard to the 'Economic - Performance per sector' chart illustrated in Figure 2.C, we can see all sectors that belong to the 'DRAGOUMI I.' street. This chart features the economic performance of a sector, which is shown as a percentage on the $\mathrm{Y}$ axis, and the yearly income for each sector, depicted as a disk with variable size. Right next to this chart there is another chart that shows the number of parking spots per sector. We can see that two sectors, namely 21.324 and 21.184 have the lowest performance and also low yearly incomes while spanning a low number of spaces. Comparing the two sectors we can safely assume that sector 21.324 can be switched to a Resident type, minimizing the loss of income and parking spaces while also tackling with the negative comments of the citizens about parking. The entire process can be repeated if there is a need to allocate more parking spots on residents, or vice versa.

\subsection{Evaluation Study}

A number of 27 end-users from the Municipality of Thessaloniki used the aforementioned scenario to test and evaluate the platform. As a result of the evaluation workshops, a great deal of information was gathered on the usability and practicalities of the dashboard. The difficulties that users experienced were recorded as part of their responses questionnaires.

More specifically, our platform was evaluated positively on its usefulness and on allowing faster decisions, assisting to the accomplishment of new tasks and encouraging the combination of different types of data (economic, environmental and social) for decision making. The contribution of the platform that was 
evaluated most positively by the users was the fact that more parameters than before are now considered before making a decision. Most of the users think that the visualizations of the platform are helpful in extracting the information they need for decision making. Many users think that the use of effective filtering is absolutely necessary to make the features easier to understand and to use.

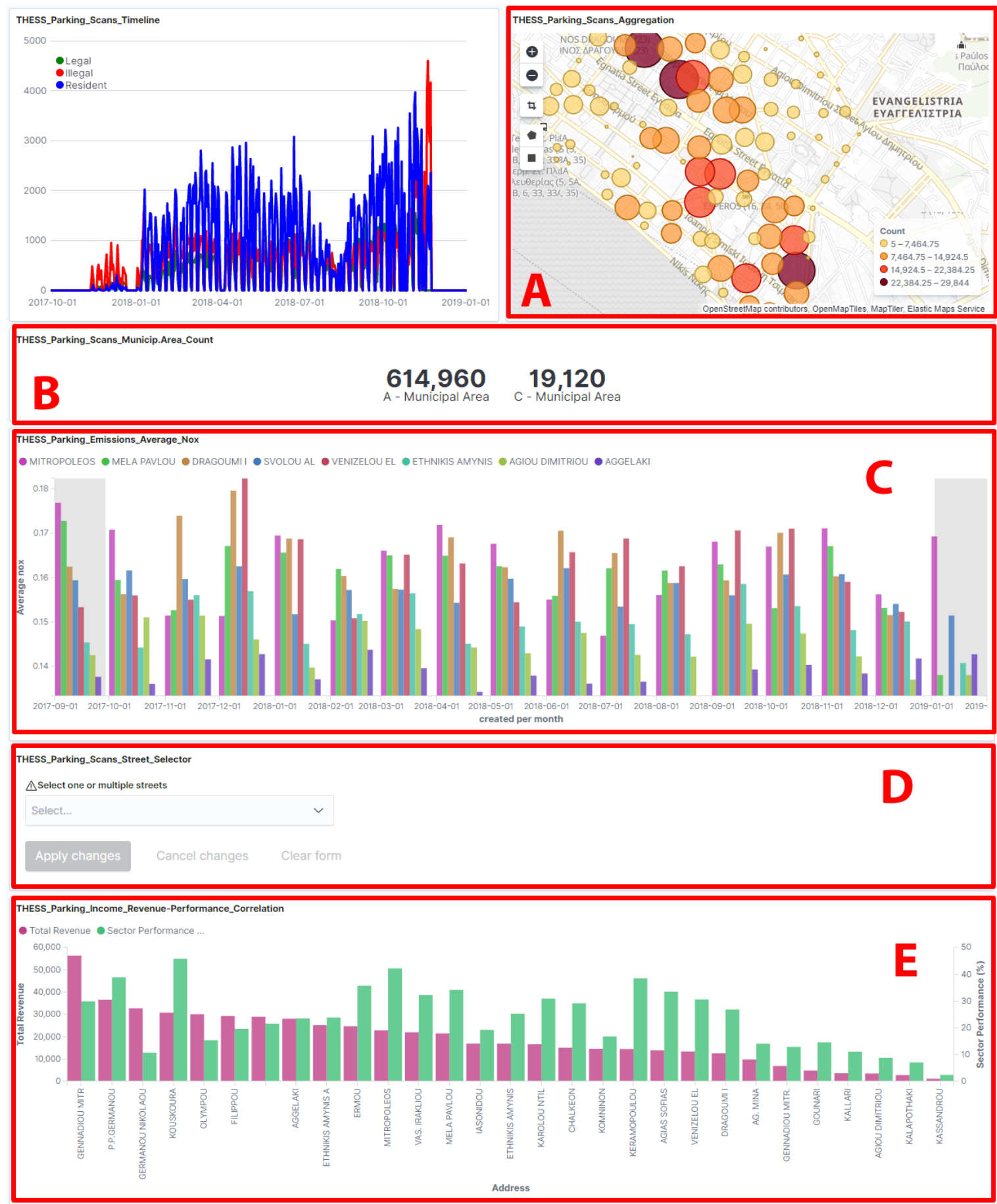

Figure 1. Dashboard showing: A) Parking Scans aggregation clustering, B) Parking Scans Municipality Area Count, C) Emission Data, D) Street Selector component, E) Parking Income Revenue/Performance correlation 


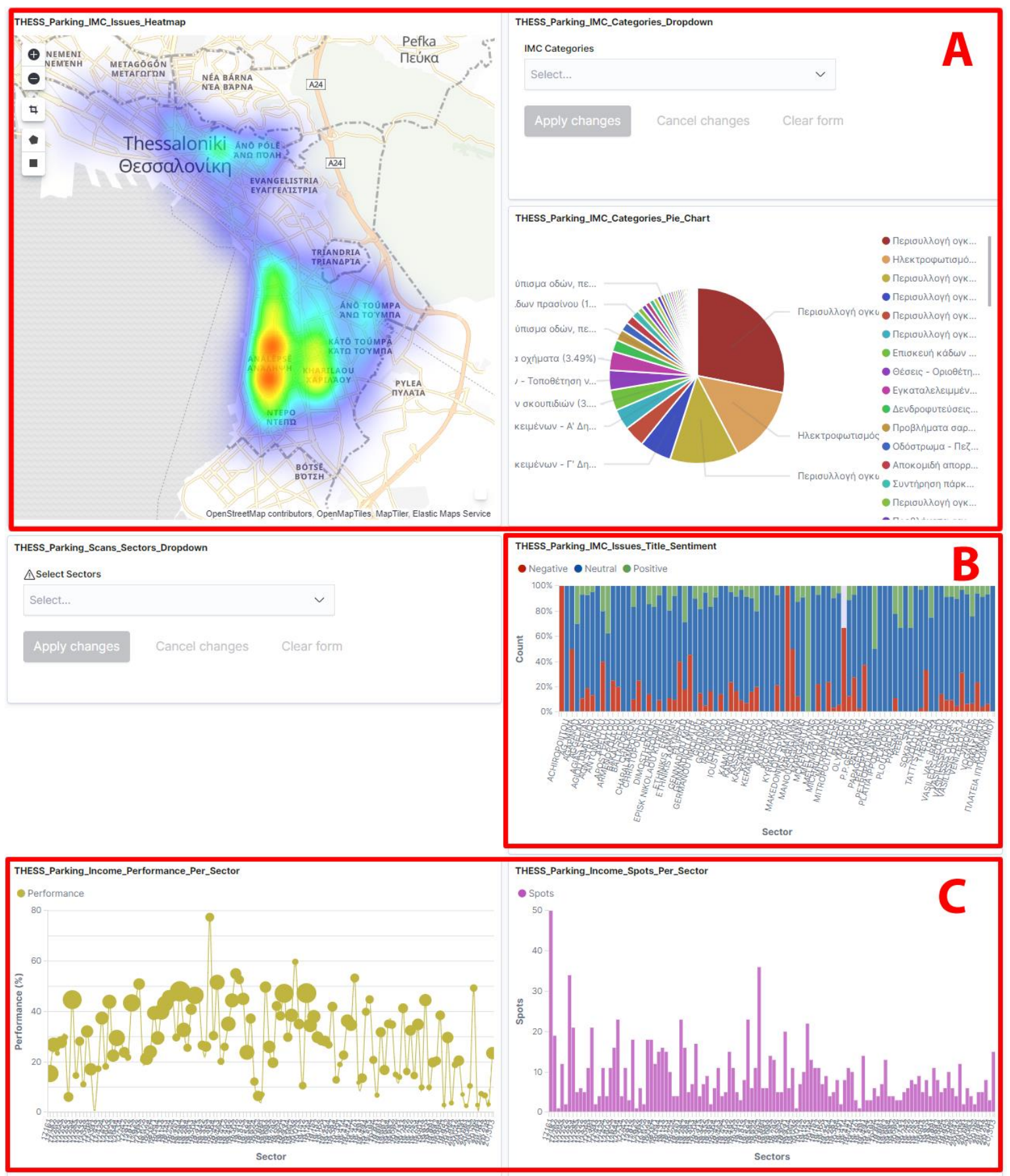

Figure 2. Dashboard showing: A) ImproveMyCity charts, B) Sentiment bar chart, C) Parking Income/Performance and Spots per Sector charts

Concerning the dataset included in the current version of the dashboard the users evaluated positively the quality and quantity of the visualized data but also made a series of suggestions. Many users would like more charts to be included in the environmental section, and specifically more data on air quality. They also suggested that the maps should include more GIS data on land use, concerning all aspects of economic life like tourism and commerce, and cover more policy fields. They would find useful information of how busy a street is commercial-wise, whether there are hospitals or public service buildings close to specific sectors, or if they knew the number of residential buildings of a street. Other users would like more social data 
(complaints/suggestions) coming from the permanent residents to be included. A few users would find it useful to visualize data on the duration of the parking sessions. Finally, regularly updated or live data would also be useful for optimal patrol calculation and improvement of supervision.

\section{CONCLUSIONS}

In the use case of Thessaloniki's controlled parking system, our work has helped to balance between economic growth, environmental impact and citizen's satisfaction, by harmonizing and visualizing heterogenous sources of data, effectively shifting the paradigm of policy making from intuition-based to evidence-driven. In evaluating our work a multi-step process was designed that involved user recruitment, user training, formulation of platform-usage scenarios, platform evaluation based on questionnaires, and finally in-depth questionnaire analysis. Most of the end-users found useful the dashboards and recognized their potential in supporting informed decisions and enabling evidence-based policy making. The use of heterogeneous data sources and the visualization of environmental, social and economic evidence in the same dashboard were considered important contributions of the platform, which have the potential to lead in more effective and accurate decisions combining and balancing different information, which is the cardinal objective of our work.

\section{ACKNOWLEDGEMENT}

The research leading to these results has received funding from the European Community's Horizon 2020 - Research and Innovation Framework Programme under grant agreement no 770469, CUTLER.

\section{REFERENCES}

CUTLER Deliverable 2.2. Threat analysis for policy-supporting hybrid cloud infrastructure. Currently not available for retrieval.

CUTLER Deliverable 3.2. First version of the framework for the collection, cleaning, integration and anonymization of big data. Retrieved from https://www.cutler-h2020.eu/download/520

CUTLER Thessaloniki Pilot, Kibana Dashboard. Retrieved 20 March 2020, from https://pilots.cutler-h2020.eu:5603/

Dror, Y. (2017). Public policy making reexamined. Routledge.

Dunn, W. N. (1981). Public Policy Analysis Englewood Cliffs. N.].: Prentice-Hall.

Elasticsearch, The heart of the Elastic Stack. Retrieved 20 March 2020, from https://www.elastic.co/elasticsearch

Improve My City - Municipality of Thessaloniki, Direct citizen-government communication \& collaboration. Retrieved 20 March 2020, from https://imc.thessaloniki.gr/imc

Kibana, Your window into the Elastic Stack. Retrieved 20 March 2020, from https://www.elastic.co/kibana

Sanderson, I. (2002). Evaluation, policy learning and evidence-based policy making. Public administration, 80(1), 1-22.

THESi, Municipality of Thessaloniki Controlled Parking System. Retrieved 20 March 2020, from https://www.thesi.gr

Treasury, H. M. S. (2000). Spending review: public service agreements.

Tsakalidis, A., Papadopoulos, S., Voskaki, R., Ioannidou, K., Boididou, C., Cristea, A. I., ... \& Kompatsiaris, Y. (2018). Building and evaluating resources for sentiment analysis in the Greek language. Language resources and evaluation, 52(4), 1021-1044.

Tsampoulatidis, I., Ververidis, D., Tsarchopoulos, P., Nikolopoulos, S., Kompatsiaris, I., \& Komninos, N. (2013, October). ImproveMyCity: an open source platform for direct citizen-government communication. In Proceedings of the 21st ACM international conference on Multimedia (pp. 839-842).

Turf.js, Advanced geospatial analysis for browsers and Node.js. Retrieved 20 March 2020, from https://turfjs.org/ 